\title{
Collision Models in the Hawk DSMC Implementation
}

\author{
July 1, 1996 \\ Sergey Gimelshein, \\ Gennady Markelov, \\ Institute for Theoretical and Applied Mechanics, Novosibirsk, Russia \\ Marc Rieffel ${ }^{1}$, \\ Scalable Concurrent Programming Laboratory, California Institute of Technology
}

\begin{abstract}
This paper presents a series of tests that have been conducted to validate the collision models of the HawkDSMC implementation. These tests cover the hard sphere (HS), variable hard sphere (VHS), and variable soft sphere (VSS) collision models, and the LarsenBorgnakke energy exchange model, for non-reacting gas mixtures. The results show that Hawkobtains the correct results in all cases under consideration.
\end{abstract}

\section{Introduction}

Recent advances in microprocessor performance have been driven primarily by improvements in manufacturing technology. New processes and equipment have paved the way for smaller feature sizes and larger die sizes. These have in turn enabled the production of microprocessors with more transistors, operating at lower voltages and higher clock rates. One of the key pieces of equipment in microelectronics manufacturing is the plasma reactor, used in 30 to 40 percent of the processing steps. Plasma reactors use energetic rarefied gases, plasmas, to remove particles from, and deposit particles on, silicon wafers. Improving the design of these reactors, and the processes that they are used for, will enable the microelectronics industry to make smaller, cheaper, and faster microprocessors.

Design and optimization of plasma reactors has so far been largely empirical. Experiments have been conducted to improve process configurations, but because of the high equipment and

\footnotetext{
${ }^{1}$ 256-80 Caltech, Pasadena, CA, 91125; marc@scp.caltech.edu; 818-395-3903.
} 


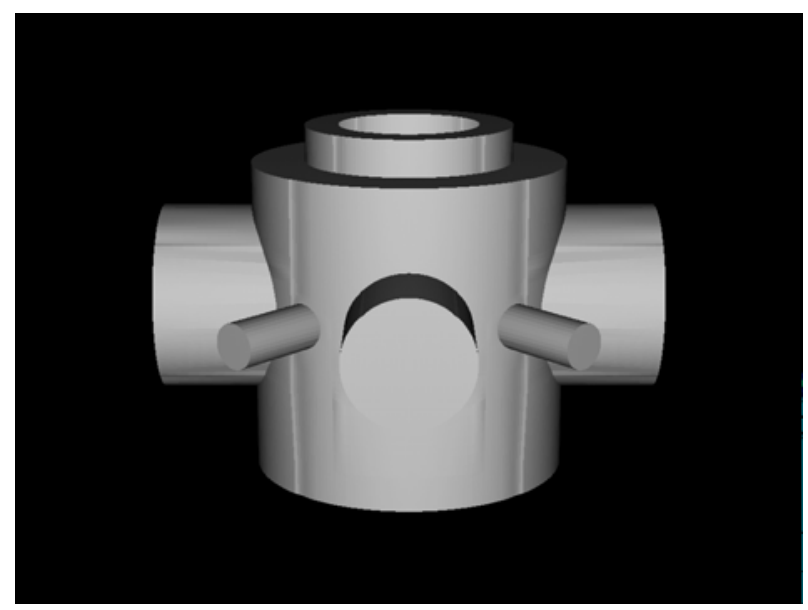

Figure 1: A Plasma Reactor

operating costs of plasma reactors, detailed parametric studies have been economically impractical. Computer-based simulation of the plasma flow inside a reactor will allow manufacturers to evaluate the viability of different reactor designs before they are implemented. Once a reactor has been installed, simulation results will also be useful for studying the effects of different operating conditions, thereby optimizing processing stages.

Figure 1 shows a typical plasma reactor, the GEC Reference Cell, and Figure 2 schematically depicts its operation. A silicon wafer is attached to an electrode, and plasma fills the space between the wafer and another electrode. Gas flows in, reactions take place within the gas and on the surface of the wafer, and the products of these reactions are pumped out of the reactor. Electromagnetic fields, applied through the electrodes, add energy to the system.

Advanced plasma simulation capabilities will be directly applicable to problems in the microelectronics industry and can therefore have direct bearing on industrial competitiveness. Simulations will be useful for studying process optimization, compact model development, equipment evaluation, process control, and technology feasibility. Efficient modeling will reduce the time and cost of microelectronics development, and therefore help improve the quality of the next generation of microprocessors. 


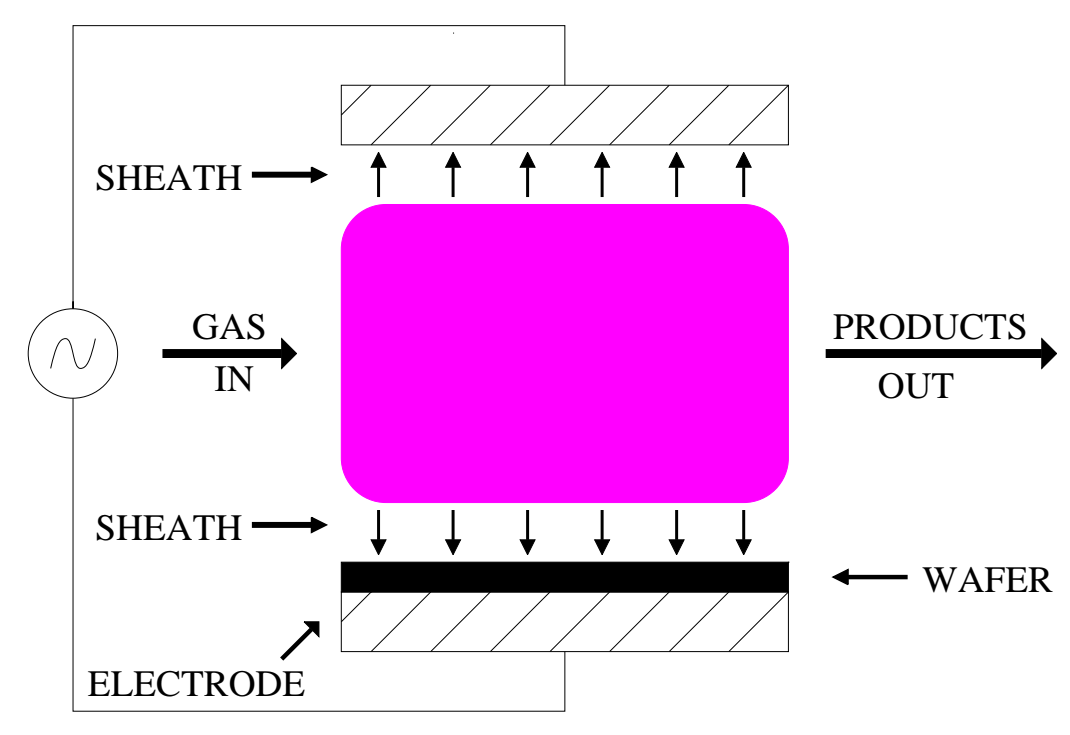

Figure 2: Reactor Schematic

\section{Direct Simulation Monte Carlo}

Plasma flow is in the transition regime: the mean free path of particles is too large for traditional continuum CFD methods to be applicable, but because collisions are important, free-molecular simulations are not appropriate, either. The Direct Simulation Monte Carlo (DSMC) method (See, for example, [Bird94].) is a widely used numerical approach for solving rarefied gas dynamics problems in the transitional regime. It simulates individual particles as they move through space and collide with solid objects and other particles. Macroscopic properties, such as density and temperature, can be computed by appropriate averaging of particle masses, positions, and velocities. Surface properties are calculated from the momentum and energy exchanges during collisions with surfaces.

In a DSMC simulation, a physical region of interest is decomposed into a number of cells. The cells are initially filled with simulation particles according to density, temperature, and velocity specifications. The simulation takes discrete steps in time, during which these particles are allowed to move throughout the domain and collide with other particles. Figure 3 shows several cells (in two dimensions) and some of the possible operations that can take place on a particle during a timestep. For example, particles may flow into the domain through injection cells, or out of the domain through exhaust cells. They may also collide with, or become embedded in, solid objects in the domain. 


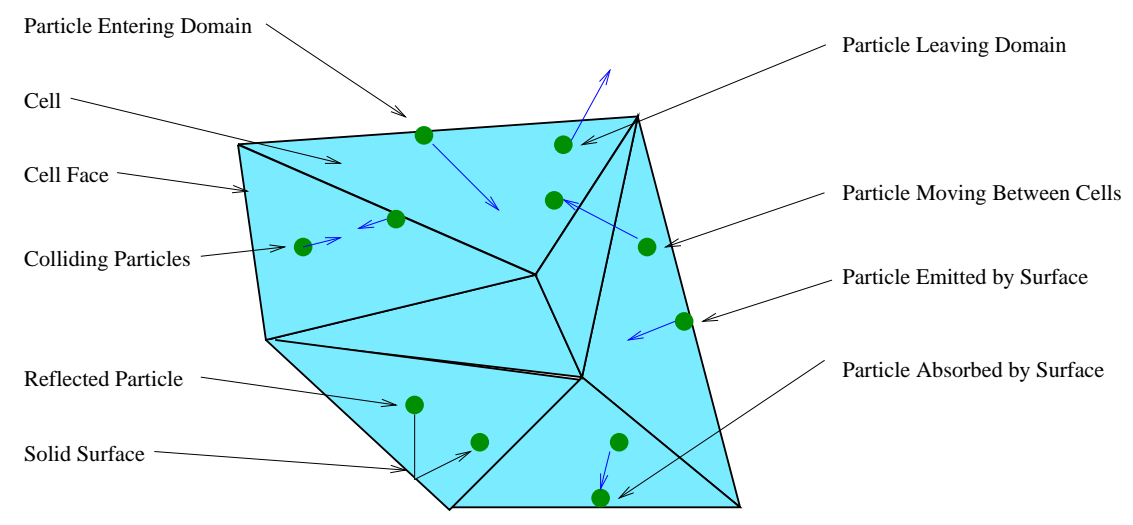

Figure 3: DSMC Cells and Particles

The DSMC method simulates the Boltzmann equation by decoupling particle movement and collisions. The two main components of any DSMC implementation are therefore the transport and collision algorithms. Particle transport is conceptually straightforward, and is discussed in detail in [Rieffe195]. Both the power and computational cost of the DSMC method are due to the collision process, and are therefore the subject of this paper.

The main goal of this work is to validate the collision models that have been implemented in Hawk, a DSMC application designed for the simulation of plasma reactors. General characteristics of Hawk, including methods for computing macroscopic parameters, grid issues, and the parallel implementation, are discussed in [Rieffe195]. The main purpose of this paper is to present the collision models that are implemented in Hawk, and to demonstrate their validation.

For the purposes of validation, it has been useful to compare the results obtained with Hawkto those obtained with another DSMC implementation, SMILE. SMILE is a Computational tool for solving problems of rarefied gas aerodynamics [Ivanov92], created at the Institute of Theoretical and Applied Mechanics (Novosibirsk, Russia). It is based on the majorant principle of construction and substantiation of numerical schemes for the DSMC method. The coupling of "cell" and "free cell" schemes [Ivanov94] provides the required spatial resolution throughout the whole flow field, including regions with strong gradients. The preprocessing subsystem of this tool is used for defining the geometric model of a space vehicle and for specifying boundary and initial conditions. The results of a computation, both flow fields and distributed surface characteristics, can be analyzed with the postprocessing system.

The following sections describe the collision models that have been implemented in Hawk, 
and present results of a series of validation tests, comparing against analytical results as well as the results of SMILE. 


\section{DSMC Collision Overview}

Generally, the global problem of creating collision models for DSMC method may be divided into several subproblems, related to creation of

- intermolecular potential models

- models of rotational degrees of freedom of molecules

- models of vibrational degrees of freedom of molecules

- models of chemical reactions.

The basic models for all four groups used in DSMC method are presented below.

\subsection{The Lennard-Jones Potential}

A number of models to describe intermolecular potential is suggested in references. It is usually assumed that the potential function includes molecular attraction at large distances and repulsion at small ones. The most famous and mathematically convenient potential, taking into account molecular attraction and repulsion, is the Lennard-Jones potential,

$$
\phi(r)=4 \varepsilon\left[\left(\frac{\sigma}{r}\right)^{12}-\left(\frac{\sigma}{r}\right)^{6}\right],
$$

where $\sigma$ is the distance at which the potential function changes its sign, $\varepsilon$ is the minimum potential value, and $r$ is the distance between the particle centers. The deflection angle, $\chi$, is,

$$
\chi=\pi-2 \int_{0}^{W_{1}}\left(1-W^{2}-\frac{\phi}{\frac{1}{2} m_{R} g^{2}}\right)^{-\frac{1}{2}} d W
$$

where $W_{1}>0$ is the root of equation,

$$
1-W^{2}-\frac{\phi}{\frac{1}{2} m_{R} g^{2}}=0
$$

$W=b / r$, and $b$ is the distance of closest approach of the undisturbed molecular trajectories in the center of mass frame of reference.

In dimensionless variables, $\chi$ may be rewritten as

$$
\chi=\pi-2 W_{1} \int_{0}^{1}\left[1-\left(W W_{1}\right)^{2}-\frac{1}{6}\left(\frac{W W_{1}}{W_{0}}\right)^{12}+\frac{1}{3}\left(\frac{W W_{1}}{W_{0}^{\prime}}\right)^{6}\right]^{-\frac{1}{2}} d W
$$


where $W=\frac{b^{\star}}{r^{\star}}, W_{0}=b^{\star}\left(\frac{g^{\star}}{24}\right)^{\frac{1}{12}}, W_{0}^{\prime}=b^{\star}\left(\frac{g^{\star}}{12}\right)^{\frac{1}{6}}, b^{\star}=\frac{b}{\sigma}, r^{\star}=\frac{r}{\sigma}$, and $g^{\star}=\frac{m_{R} g^{2}}{2 \varepsilon}$.

In statistical simulation, the dependence $\chi$ on two parameters $W_{0}$ and $W_{0}^{\prime}$ necessitates the use of interpolation tables for computing the maximum impact parameters and deflection angles. To find the deflection angle for a pair of colliding particles in simulation, it is necessary to determine the value $b_{\max }^{\star}$ from the interpolation tables at the specified deflection angle cut-off, sample $b^{\star}$ and, using the tables again, find $\chi$. Because of the high computational cost of this process, as well as difficulties in determining the mean free path, the Lennard-Jones potential is typically only applied to homogeneous relaxation cases and simple one-dimensional problems.

\subsection{The Inverse Power Law Potential}

A more suitable model for high-temperature flows, where intermolecular repulsion prevails, is the Inverse Power Law (IPL) model,

$$
\phi(r)=\frac{\kappa}{(\eta-1) r^{\eta-1}},
$$

where $\eta$ is the power determining the "hardness" of particles. The deflection angle is,

$$
\chi=\pi-2 \int_{0}^{W_{1}}\left[1-W^{2}-\frac{2}{\eta-1}\left(\frac{W}{W_{0}}\right)^{\eta-1}\right]^{-\frac{1}{2}} d W
$$

where $W_{0}=b\left(\frac{m_{R} g^{2}}{\kappa}\right)^{\frac{1}{\eta-1}}$, and $W_{1}>0$ is the root of equation $1-W^{2}-\frac{2}{\eta-1}\left(\frac{W}{W_{0}}\right)^{\eta-1}=0$. Note that $\chi$ is a function of one parameter, $W_{0}$, and may be found from various approximate analytical expressions [Nanbu81].

The total, viscosity, and diffusion cross-sections for IPL model are,

$$
\begin{gathered}
\sigma_{T}=\int \sigma d \Omega=\infty \\
\sigma_{\mu}=\int\left(1-\cos ^{2} \chi\right) \sigma d \Omega=2 \pi A_{2}(\nu)\left(\frac{m_{R} \kappa}{g^{2}}\right)^{\frac{2}{\eta-1}} \\
\sigma_{D}=\int(1-\cos \chi) \sigma d \Omega=2 \pi A_{1}(\nu)\left(\frac{m_{R} \kappa}{g^{2}}\right)^{\frac{2}{\eta-1}} .
\end{gathered}
$$

To use the IPL model in a DSMC simulation, it is necessary to limit $\sigma_{T}$. This can be achieved by specifying either a deflection angle cut-off or a maximum impact parameter value. In most 
cases, the latter is chosen. Since $\chi$ is a function of $W_{0}$, the deflection angle cut-off may be applied through the specification of a maximum value $W_{0, m}$ of $W_{0}$. This yields,

$$
\sigma_{T}=\pi W_{0, m}^{2}\left(\frac{\kappa}{m_{R} g^{2}}\right)^{\frac{2}{\eta-1}}
$$

At $\eta=\infty$, the IPL model is equivalent to the hard sphere model for,

$$
\sigma_{T}=\pi d^{2}, \quad b=d \cos (\chi / 2)
$$

where $d$ is the molecular diameter. 


\section{The Hard Sphere Collision Model}

Earlier versions of this implementation have been validated by comparison with published results [Zhong95] for heat-transfer problems [Rieffe195]. This section presents several further tests, using the Hard Sphere (HS) collision model for gas mixtures and more complicated geometries.

\subsection{Box Tests}

The first series of tests considered here was designed to verify that all of the basic DSMC operations were correctly implemented, including transport, inflow, accomodation, and hard sphere (HS) collisions. This series was performed in a 12-cell uniform cubic grid, with all walls specular.

The first test considered Ar and Ar*, two identical species. It was verified that the collision frequencies were correct. Macroparameters were observed to be identical for both species.

The second test considered Ar and He in equal concentrations, with density $n=1.83 \times 10^{19}$ particles $/ \mathrm{m}^{3}$, and both species at a temperature of $300 \mathrm{~K}$. The system remained in equilibrium, and all collision counts were equal to theoretical values.

The next test considered a mixture of Argon and Helium at different temperatures. The system reached equilibrium of $T_{A r}=T_{H e}=300 \mathrm{~K}$, the collision numbers were correct, and the system reached equilibrium at the same rate as SMILE.

The next test considered different concentrations of Ar and $\mathrm{He},[\mathrm{Ar}]=0.9,[\mathrm{He}]=0.1 . T_{A r}=$ $100 \mathrm{~K} T_{H e}=500 \mathrm{~K}$. Verified that the final temperature for both species was $0.9 * 100+0.1 * 500=$ $140 \mathrm{~K}$.

The last box case considered the opposite concentrations, $[\mathrm{Ar}]=0.1,[\mathrm{He}]=0.9$, with $T_{A r}=$ $100 \mathrm{~K}$ and $T_{H e}=500 \mathrm{~K}$. The final temperature for both species was the correct value, $0.1 \times 100+$ $0.9 \times 500=460 \mathrm{~K}$.

\subsection{Cylinder Test}

The final HS test considers Mach-4 flow past a cylinder. The cylinder has a radius of $0.05 \mathrm{~m}$, and the grid extends $0.15 \mathrm{~m}$ in front of the center of the cylinder, $0.35 \mathrm{~m}$ behind the center of the cylinder, and $0.2 \mathrm{~m}$ to the side of the cylinder. The freestream density is $1.83 \times 10^{21}$ particles per 
cubic meter, the freestream speed is $526.86 \mathrm{~m} / \mathrm{s}$, and the freestream temperature is $50 \mathrm{~K}$. The cylinder itself is fully accomodating at $300 \mathrm{~K}$. The inflow is composed of $50 \% \mathrm{Ar}$ and $50 \% \mathrm{He}$. Because the symmetric nature of the problem, only the upper half of the cylinder was simulated. A thin $(0.01 \mathrm{~m})$ 3-D grid was used for Hawk, while a 2D grid was used for SMILE. Argon density plots for both codes are shown in Figures 4 and 5. The results of the simulations agree very well. 


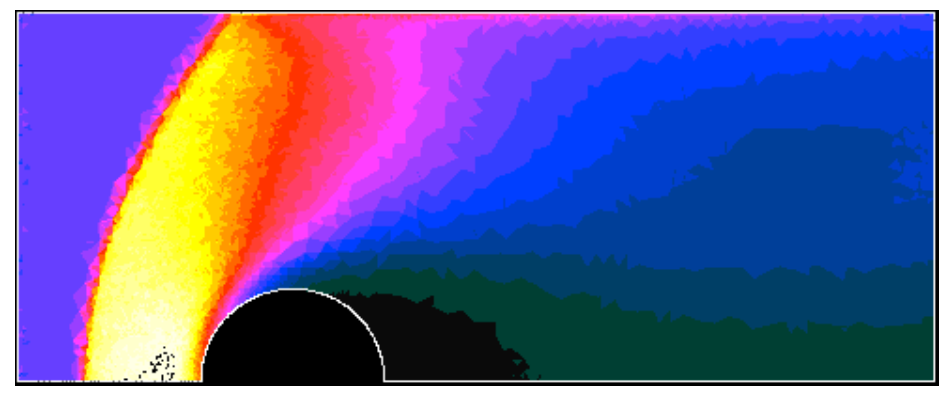

Figure 4: HawkAr Density

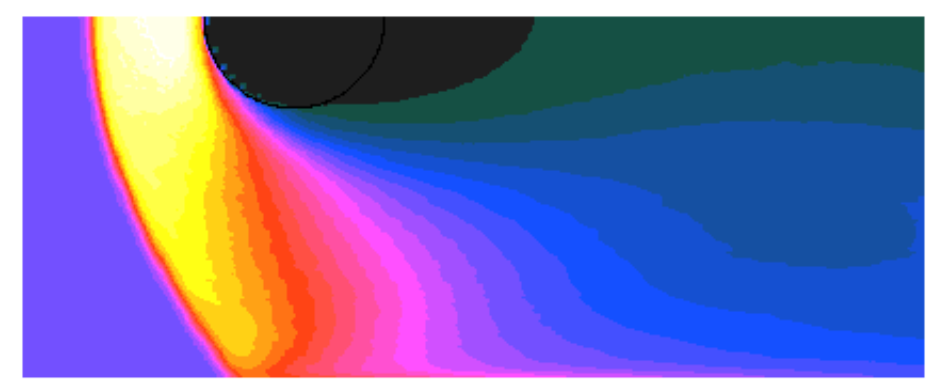

Figure 5: SMILE Ar Density 


\section{The Variable Hard Sphere Collision Model}

The Variable Hard Sphere (VHS) model, developed by Bird in 1981, uses cross sections that are functions of relative velocity, but with hard sphere (HS) scattering angles. Collision, viscosity, and diffusion cross sections therefore take the form,

$$
\begin{aligned}
\sigma_{T} \equiv \pi d^{2} & =c\left(m_{R} g^{2} / 2\right)^{-\alpha} \\
\sigma_{\mu} & =\frac{2}{3} \pi d^{2} \\
\sigma_{D} & =\pi d^{2} .
\end{aligned}
$$

where $c$ and $\alpha$ are parameters of the model. As in the HS model, the scattering angle is given by,

$$
b=d \cos (\chi / 2) \text {. }
$$

Equating $\sigma_{\mu}$ for the IPL and VHS models yields,

$$
\alpha=\frac{2}{\eta-1}, \quad c=3 \pi A_{2}(\nu)(\kappa / 2)^{\alpha} .
$$

For a gas at equilibrium, $\sigma_{T}$ may be written as,

$$
\sigma_{T}=\sigma_{r e f}\left(\frac{T_{r e f}}{T}\right)^{\alpha}
$$

where $\sigma_{r e f}$ is the reference value of the cross-section at the reference temperature $T_{\text {ref }}$.

The VHS model is currently the most popular for DSMC simulations, because of its simplicity and its good approximation to real intermolecular potentials. Note also that VHS collisions are much more "efficient" than IPL collisions. For example, relaxation for Maxwellian molecules under the IPL model, with the deflection angle cut-off $1^{\circ}$, is about six times slower than under the VHS model with the same viscosity-temperature dependence.

In some cases, the parameters $\alpha, \sigma_{r e f}$, and $T_{r e f}$ may be known for collisions between particles of a species A, and similarly for a species B, but not for collisions between species A and B. In this case, they can be approximated according to the formulae,

$$
\begin{gathered}
\alpha_{A B}=\frac{\alpha_{A}+\alpha_{B}}{2}, \\
\sigma_{A B}=\left(\frac{\sqrt{\sigma_{A}}+\sqrt{\sigma_{B}}}{2}\right)^{2}, \\
T_{A B}=\frac{T_{A}+T_{B}}{2} .
\end{gathered}
$$




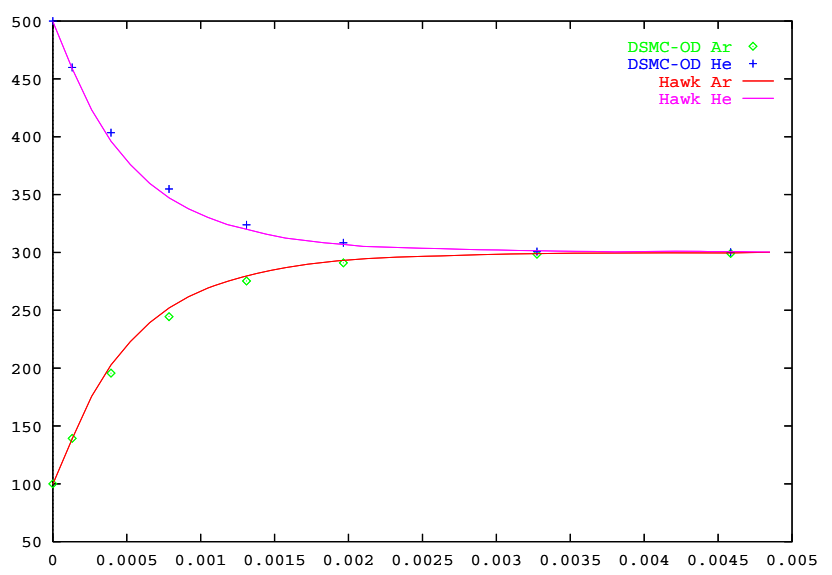

Figure 6: VHS 50-50

\subsection{Box Tests}

Initial validation of the VHS model consisted of running a uniform specular box and comparing the number of collisions between Hawkand SMILE. The results were in excellent agreement. The next series of tests studied the temperature relaxation rate in the specular box, with mixtures of Argon and Helium at different temperatures and in different concentrations.

The first of these tests was a 50\%-50\% mixture of Argon and Helium, with Argon at 100 $\mathrm{K}$ and Helium at $500 \mathrm{~K}$. As would be expected, the final temperature of the mixture was $300 \mathrm{~K}$. Figure 6 shows that SMILE and Hawkboth reached the correct final temperature at the same rate.

The second test was with $90 \%$ Argon at $100 \mathrm{~K}$ and $10 \%$ Helium at $500 \mathrm{~K}$. The number of collisions, and convergence rates, as shown in Figure 7, both agreed.

\subsection{Cylinder Test}

The final test of the VHS model was the cylinder problem, as described in Section 4.2. For this, we used a mixture of $50 \%$ Argon and $50 \%$ Helium, with $\alpha=0.5$. Density plots for the two codes are shown in Figures 8 and 9, and these agree very well. The density along the streamline was also compared and found to be the same for the two codes. 


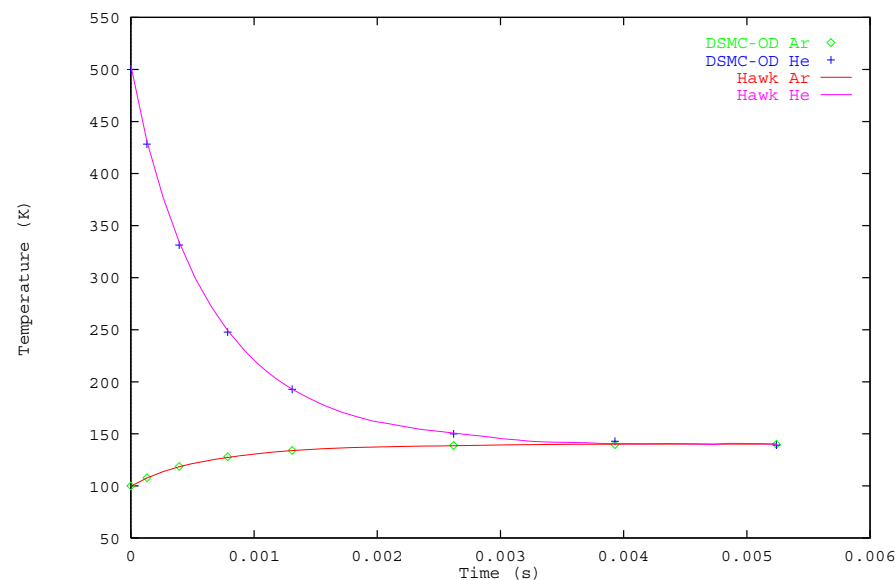

Figure 7: Ar-He 90-10 VHS 


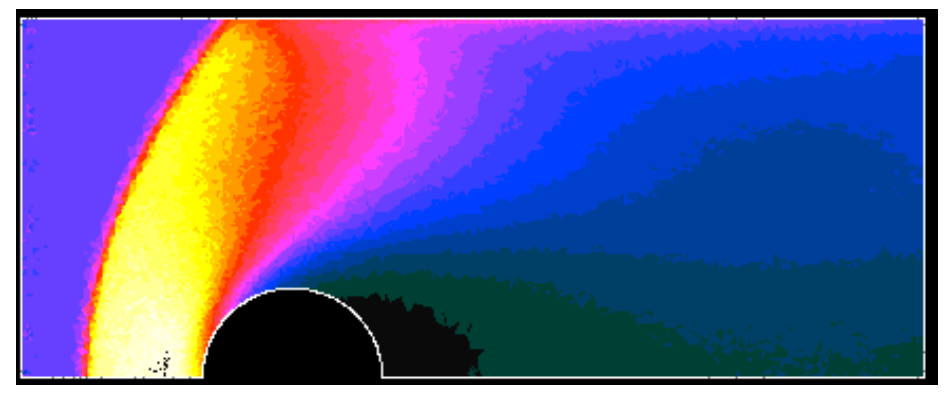

Figure 8: HawkAr Density

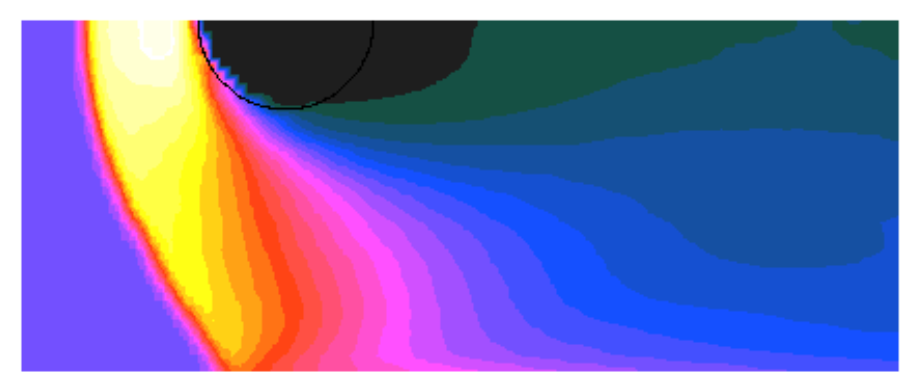

Figure 9: SMILE Ar Density 


\section{The Variable Soft Sphere Collision Model}

The main disadvantage of the VHS model arises when equating viscosity coefficients $\sigma_{\mu}$ for the VHS and IPL models. The diffusion cross-sections for the two models, and hence, the diffusion coefficients, coincide only for the hard sphere model. The variable soft sphere (VSS) model developed by Koura (1991) has no such drawback. In this model, the primary equations are,

$$
\begin{gathered}
\sigma_{T} \equiv \pi d^{2}=c^{\prime}\left(\frac{1}{2} m_{R} g^{2}\right)^{-\alpha} \\
\sigma_{\mu}=\frac{6 \psi}{(\psi+1)(\psi+2)} \frac{2}{3} \pi d^{2} \\
\sigma_{D}=\frac{2}{\psi+1} \pi d^{2} \\
b=d \cos ^{\psi}(\chi / 2) .
\end{gathered}
$$

Equating the VSS and IPL parameters, we obtain,

$$
\begin{gathered}
\alpha=\frac{2}{\eta+1}, \\
\psi=\left(\frac{A_{1}(\eta)}{A_{2}(\eta)}-\frac{1}{2}\right)^{-1}, \\
c^{\prime}=3 \pi A_{2}(\eta)(\kappa / 2)^{\alpha} \frac{(\psi+1)(\psi+2)}{6 \psi} .
\end{gathered}
$$

In a collision, we define two scattering angles, $\chi$ and $\epsilon . \quad \chi$ is the angle between the precollision relative velocity and the post-collision relative velocity. $\epsilon$ is the azimuthal impact angle measured between the collision plane and some reference plane. For hard sphere collisions (HS and VHS), the scattering angles $\chi$ and $\epsilon$ are both distributed uniformly. In terms of the molecule diameter $d$ and the impact parameter $b$, we can write

$$
\frac{b}{d}=\cos \left(\frac{\chi}{2}\right)
$$

For VSS colisions, we use a parameter $\beta$ to characterize the anisotropy of the scattering angle, and can therefore write,

$$
\left(\frac{b}{d}\right)^{\beta}=\cos \left(\frac{\chi}{2}\right) .
$$

These parameters must be used for computing the post-collision relative velocity $\overrightarrow{g^{\prime}}$ as a function of the pre-collision relative velocity $\overrightarrow{g_{0}}$ and the post-collision relative speed $g^{\prime}$. For elastic 
collisions, $g^{\prime}$ is simply the magnitude of $\vec{g}$, while for inelastic collisions it may be different. The procedure for computing $\overrightarrow{g^{\prime}}$ is as follows.

First, the azimuthal impact angle $\epsilon$ is computed using a random number $R_{1}$,

$$
\epsilon=2 \pi R_{1}
$$

Second, the scattering angle $\chi$ is computed using another random number $R_{2}$,

$$
\begin{gathered}
\cos \chi=2 R_{2}^{\beta}-1 \\
\sin \chi=\sqrt{1-\cos ^{2} \chi} .
\end{gathered}
$$

Next, the pre-collision relative velocity $\overrightarrow{g_{0}}$ is scaled to have the magnitude of the post-collision relative speed,

$$
\vec{g}=g^{\prime} \frac{\overrightarrow{g_{0}}}{\left|\overrightarrow{g_{0}}\right|} .
$$

The components of $\overrightarrow{g^{\prime}}$ can then be computed as

$$
\begin{gathered}
g_{x}^{\prime}=g_{x} \cos \chi+\sqrt{g_{y}^{2}+g_{z}^{2}} \sin \chi \sin \epsilon \\
g_{y}^{\prime}=g_{y} \cos \chi+\sin \chi\left(g^{\prime} g_{z} \cos \epsilon-g_{x} g_{y} \sin \epsilon\right)\left(g_{y}^{2}+g_{z}^{2}\right)^{-1 / 2} \\
g_{z}^{\prime}=g_{z} \cos \chi-\sin \chi\left(g^{\prime} g_{y} \cos \epsilon+g_{x} g_{z} \sin \epsilon\right)\left(g_{y}^{2}+g_{z}^{2}\right)^{-1 / 2}
\end{gathered}
$$

\subsection{Test 1}

The first VSS test case was the box problem with Helium and Argon parameters as shown in the table below. The number of collisions was compared with SMILE. The system was shown to stay at equilibrium at $100 \mathrm{~K}$.

\begin{tabular}{|l|r|r|r|l|}
\hline Species & Ar-Ar & Ar-He & He-He & \\
\hline$\alpha$ & 0.31 & 0.235 & 0.16 & \\
\hline$\beta$ & 0.714 & 0.754 & 0.794 & \\
\hline Fraction & 0.5 & & 0.5 & \\
\hline Temperature & 100 & & 100 & $\mathrm{~K}$ \\
\hline
\end{tabular}




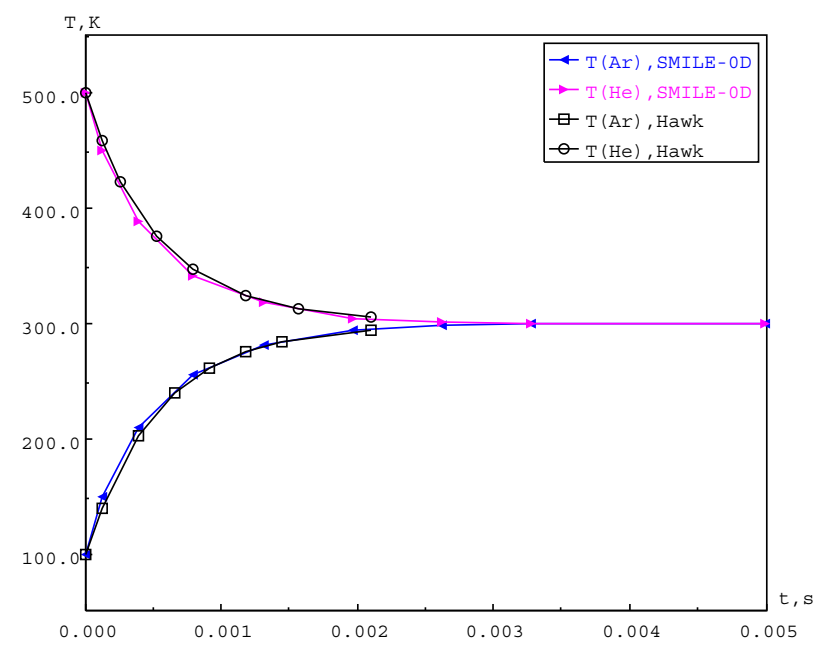

Figure 10: VSS 50-50

\subsection{Test 2}

The next test for the VSS model was with Argon and Helium at different initial temperatures, as shown in the table below. The convergence rates of the temperatures were shown to agree with the results of SMILE, as shown in Figure 10.

\begin{tabular}{|l|r|r|r|r|}
\hline Species & Ar-Ar & Ar-He & He-He & \\
\hline$\alpha$ & 0.31 & 0.235 & 0.16 & \\
\hline$\beta$ & 0.714 & 0.754 & 0.794 & \\
\hline Fraction & 0.5 & & 0.5 & \\
\hline Temperature & 100 & & 500 & $\mathrm{~K}$ \\
\hline
\end{tabular}

\subsection{Test 3}

The final box test for the VSS model used Argon and Helium in different concentrations, according to the table below. Results were in close agreement with those of SMILE, as shown in Figure 12.

\begin{tabular}{|l|r|r|r|r|}
\hline Parameter & Ar-Ar & Ar-He & He-He & \\
\hline$\alpha$ & 0.31 & 0.235 & 0.16 & \\
\hline$\beta$ & 0.714 & 0.754 & 0.794 & \\
\hline Fraction & 0.9 & & 0.1 & \\
\hline Temperature & 100 & & 500 & $\mathrm{~K}$ \\
\hline
\end{tabular}




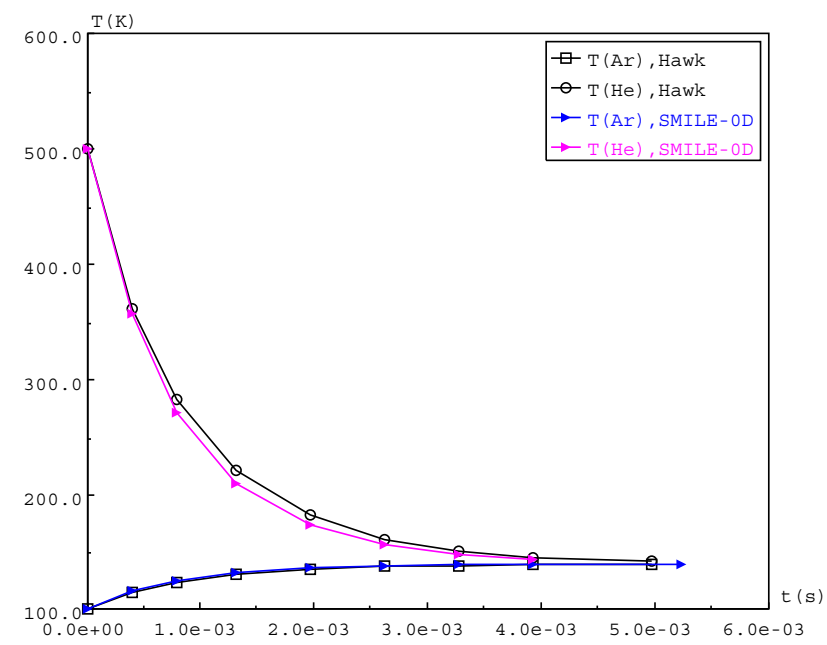

Figure 11: VSS 90-10

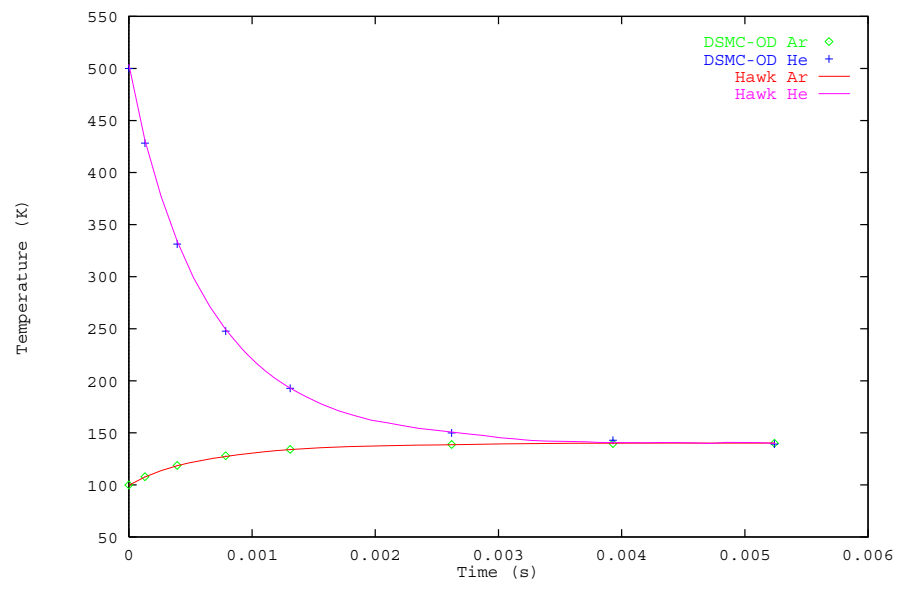

Figure 12: VSS 90-10 


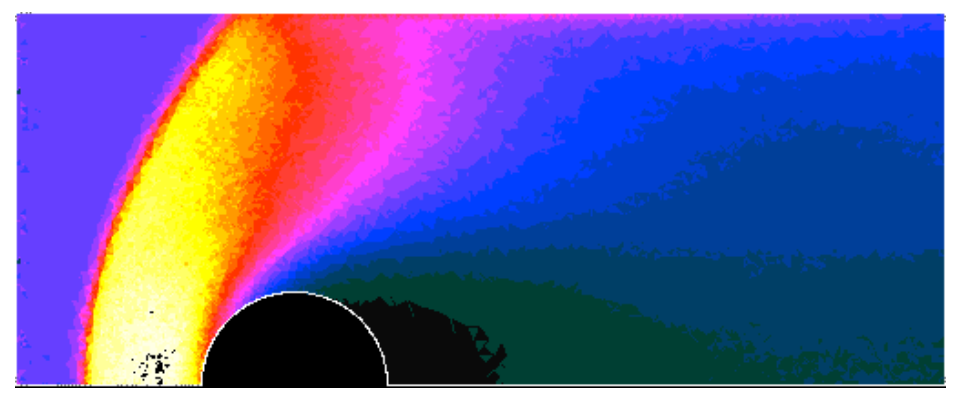

Figure 13: HawkAr Density

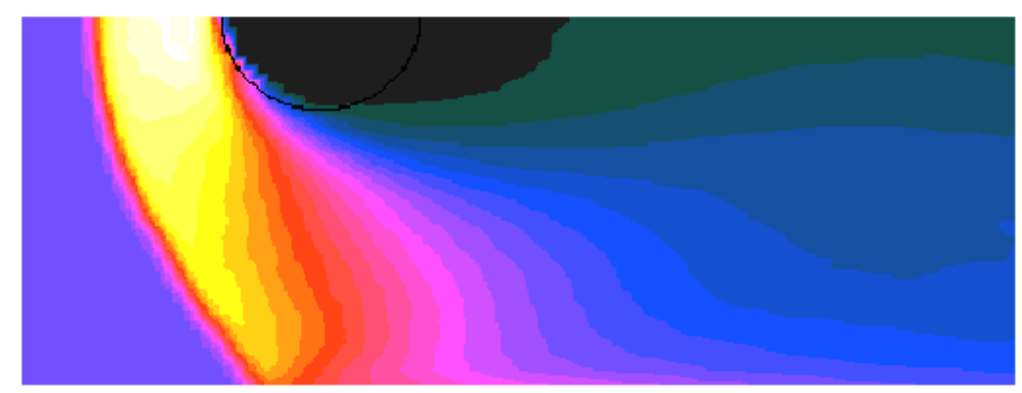

Figure 14: SMILE Ar Density

\subsection{Cylinder}

The final VSS test considered the cylinder problem, as described in 4.2. Results for Hawkand SMILE are shown in Figures 13 and 14. 


\section{The Larsen-Borgnakke Energy Exchange Model}

The Larsen-Borgnakke model of energy exchange is used to describe internal energy modes for rotation and vibration[Borgnakke75]. Relative translational and internal post-collision energies of colliding particles are assumed to be distributed according to equilibrium distribution function.

The model associates with each species a number of atoms $n_{a}$ and a characteristic vibrational temperature $\Theta_{v}$, and with each particle, rotational energy $E_{r}$ and vibrational energy $E_{v}$. Rotational and vibrational energies are assumed to be continuous.

This implementation considers two types of energy exchange, translational-rotational (TR) and translational-rotational-vibrational (TRV). Each collision has some probability $\phi_{R}$ of a TR exchange and some (smaller)probability $\phi_{V}$ of a TRV exchange.

\subsection{Internal Degrees of Freedom}

It is first necessary to characterize the number of degrees of freedom in each of the energy modes. Relative translational energy has 3 degrees of freedom. For rotational energy, the number of degrees of freedom $\xi_{r}$ is a function of the number of atoms, $n_{a}$, given by,

$$
\xi_{r}=\left\{\begin{array}{ll}
0 & n_{a}=1 \\
2 & n_{a}=2 \\
3 & n_{a} \geq 3
\end{array} .\right.
$$

The number of effective vibrational degrees of freedom can be derived from the simple harmonic oscillator (SHO) approximation. Vibrational degrees of freedom are therefore a function of the local temperature, $T$, and the species' characteristic vibrational temperature $\Theta_{v}$, given by,

$$
\xi_{v}=\frac{2 \Theta_{v} / T}{e^{\Theta_{v} / T}-1} \frac{n_{a}\left(n_{a}-1\right)}{2} .
$$

\subsection{Injection and Reflection}

When a particle is first injected into a domain whether by initial conditions, inflow, or surface emission, its initial rotational and vibrational energies must be computed. When a particle hits an accomodating surface, its internal energies must be recomputed. These values are sampled from the equilibrium distribution function for the specified temperature $T$. Internal energies, both rotational, $E_{r}$ and vibrational $E_{v}$, as functions of degrees of freedom, $\xi_{r}$ and $\xi_{v}$, are computed as 
follows. If the number of degrees of freedom is less than or equal to two, the internal energy can be computed using a single random number $R_{1}$,

$$
E=-\ln \left(R_{1}\right) \frac{\xi k T}{2} \quad \xi \leq 2 .
$$

If the number of degrees of freedom is greater than two, internal energy is sampled, using the acceptance-rejection method, from the distribution function,

$$
f(E)=\frac{1}{\Gamma(\xi / 2)}\left(\frac{E}{k T}\right)^{\xi / 2-1} e^{-E / k T} \quad \xi>2 .
$$

\subsection{Energy Redistribution During Collisions}

The two mechanisms for exchange of internal energy are between translational and rotational modes (TR), and between translational, rotational, and vibrational modes (TRV). These redistributions take place according to the equilibrium energy distribution function for a specified number of degrees of freedom, given by,

$$
f(E)=\frac{1}{\Gamma(\xi / 2)}\left(\frac{E}{k T}\right)^{\xi / 2-1} e^{-E / k T},
$$

where $f(E)$ is the probability of the occurence of energy $E, \xi$ is the number of degrees of freedom, $k$ is the Boltzmann constant, and $T$ is the temperature. For an exchange between two modes $A$ and $B$, with respective degrees of freedom $\xi_{A}$ and $\xi_{B}$, the joint distribution function is,

$$
f\left(E_{A}, E_{B}\right)=\frac{e^{-\left(E_{A}+E_{B}\right) / k T}}{\Gamma\left(\xi_{A} / 2\right)} \Gamma\left(\xi_{B} / 2\right)\left(\frac{E_{A}}{k T}\right)^{\xi_{A} / 2-1}\left(\frac{E_{B}}{k T}\right)^{\xi_{B} / 2-1} .
$$

If the total energy, $E_{t}=E_{A}+E_{B}$, is known,this can be rewritten as,

$$
f\left(E_{A}, E_{B}\right)=\frac{e^{-\left(E_{t}\right) / k T}}{\Gamma\left(\xi_{A} / 2\right)} \Gamma\left(\xi_{B} / 2\right)\left(\frac{E_{t}-E_{B}}{k T}\right)^{\xi_{A} / 2-1}\left(\frac{E_{B}}{k T}\right)^{\xi_{B} / 2-1} .
$$

To sample from this distribution, the following acceptance-rejection procedure is used. First, the values $x_{\max }$ and $f_{\max }$ are computed as

$$
\begin{gathered}
x_{\max }=\frac{1-\xi_{A} / 2}{2-\xi_{A} / 2-\xi_{B} / 2} \\
f_{\max }=\left(1-x_{\max }\right)^{\xi_{A} / 2-1} x_{\max }^{\xi_{B} / 2-1} .
\end{gathered}
$$


A random number $R_{1}$ is then used to compute

$$
\begin{gathered}
f=\left(1-R_{1}\right)^{\xi_{A} / 2-1}\left(R_{1}\right)^{\xi_{B} / 2-1} \\
y=f / f_{\max } .
\end{gathered}
$$

The values $f$ and $y$, and a random number $R_{2}$ are recomputed until $R_{2}<y$. Once this condition has been met, the energy $E_{t}$ is redistributed between the modes $A$ and $B$ using,

$$
\begin{gathered}
E_{A}=R_{1} E_{t} \\
E_{B}=E_{t}-E_{A} .
\end{gathered}
$$

Once a collision is selected to take place, exchanges may take place between translational, rotataional, and vibrational energy modes. A TR exchange takes place with proability $\phi_{R}$, and a TRV exchange takes place with probability $\phi_{V}$. The probabilities $\phi_{R}$ and $\phi_{V}$ can be obtained in two ways. They can either be specified as constants or computed as a function of species parameters and the local translational temperature.

In order to compute the probabilities as functions of temperature, three additional parameters must be stored for each species, the limiting value of the rotational relaxation number $Z_{r_{\infty}}$, the characteristic temperature of the inter-molecular potential, $T^{\star}$, and the effective excitation cross section $\sigma_{v}$. The TR exchange probability between species $i$ and $j, \phi_{R}^{i j}$, can then be computed as [Lumpkin91, Parker59],

$$
\phi_{R}^{i j}=\left(1+\frac{\xi_{r}}{4-2 \alpha}\right) \frac{1+\frac{\pi^{3 / 2}}{2}\left(\frac{T^{\star}}{T}\right)^{1 / 2}+\left(\frac{\pi^{2}}{4}+\pi\right) \frac{T^{\star}}{T}}{Z_{r_{\infty}}},
$$

where $\xi_{r}=\xi_{r}^{i}+\xi_{r}^{j}$ is the number of rotational degrees of freedom in the collision, $\alpha$ is the VHS collision parameter, and $T=\frac{T^{i}+T^{j}}{2}$ is the local translational temperature averaged between the two species.

The equation for the TRV exchange probability, $\phi_{V}^{i j}$, was obtained from an empirical fit [Millikan63] to experimental data witha high-temperature correction [Park85]. It can be written as,

$$
\begin{aligned}
\phi_{V}^{i j}= & \frac{\xi_{\text {tot }}}{\xi_{\text {tot }}-\xi_{v}}\left\{P_{c} \exp \left(A T^{-1 / 3}+B\right) \sigma_{r e f}\left[2(2-\alpha) \frac{k T_{r e f}}{m_{r}}\right]^{\alpha} \frac{2}{k T \sqrt{\pi}} \Gamma(2-\alpha)\left(\frac{2 k T}{m_{r}}\right)^{1 / 2-\alpha}\right. \\
& \left.+\frac{\sigma_{r e f} \sqrt{2}}{\sigma_{v}}\left[2(2-\alpha) \frac{k T_{r e f}}{m_{r}}\right]^{\alpha} \Gamma(2-\alpha)\left(\frac{2 k T}{m_{r}}\right)^{-\alpha}\right\}^{-1}
\end{aligned}
$$




$$
\begin{gathered}
A=F_{A} m_{r}^{1 / 2} \Theta_{v}^{4 / 3} \\
B=F_{B} m_{r}^{3 / 4} \Theta_{v}^{4 / 3}-F_{C},
\end{gathered}
$$

where $P_{c}=101325 \mathrm{~Pa} / \mathrm{Atm}$ is a pressure conversion factor, $\sigma_{r e f}$ and $T_{\text {ref }}$ are the VHS reference cross section and temperature, and $m_{r}$ is the reduced mass of the collision. The constants $F_{A}=$ $2.85 \times 10^{10} \mathrm{~K}^{-1} \mathrm{~kg}^{-1 / 2}, F_{B}=-2.11 \times 10^{15} \mathrm{~K}^{-4 / 3} \mathrm{~kg}^{-3 / 4}$ and $F_{C}=18.42$ are the constants obtained by empirical fit [Millikan63].

\subsubsection{TR Exchanges}

A TR exchange is performed as follows. The total collision energy to be redistributed is the sum of translational and rotational energies, $E_{c}=E_{t}+E_{r}$, where $E_{r}=E_{r}^{A}+E_{r}^{B}$ is the sum of the rotational energies of the two colliding particles. The first step in the exchange is to distribute the collision energy between translational and rotational energy modes. After checking the relative velocity in a collision, the distribution function is biased, so the number of relative translational degrees of freedom must be taken as $\xi_{t}=4-2 \alpha$. The number of rotational degrees of freedom is the sum of rotational degrees of freedom for the two colliding particles, $\xi_{r}=\xi_{r}^{A}+\xi_{r}^{B}$. The total collision energy is therefore first redistributed between $\xi_{t}$ and $\xi_{r}$, as described in Section 7.3, yielding $E_{t}$ and $E_{r}$, respectively.

The rotational energy is then redistributed between the two particles with rotational degrees of freedom $\xi_{r}^{A}$ and $\xi_{r}^{B}$, as described in Section 7.3, yielding $E_{r}^{A}$ and $E_{r}^{B}$, respectively. The postcollision relative velocity $g^{\prime}$ is then computed from the post-collision translational energy $E_{t}$ using the reduced mass of the collision, $m_{r}$,

$$
g^{\prime}=\sqrt{\frac{2 E_{t}}{m_{r}}} .
$$

\subsubsection{TRV Exchanges}

In a TRV exchange, the total collision energy is $E_{c}=E_{t}+E_{i}$, where $E_{t}$ is the translational energy and $E_{i}$ is the internal energy. The internal energy is in the sum of the internal energies of the two particles, $E_{i}=E_{i}^{A}+E_{i}^{B}$. The internal energy of a particle is the sum of rotational and vibrational enegies, $E_{i}^{A}=E_{r}^{A}+E_{v}^{A}$. Similarly, the number of degreees of freedom in the various energy modes are, $\xi_{t}, \xi_{i}, \xi_{i}^{A}, \xi_{i}^{B}, \xi_{r}^{A}, \xi_{r}^{B}, \xi_{v}^{A}$, and $\xi_{r}^{B}$. 
The total collision energy, $E_{c}$, is first distributed between translational and internal modes, using $\xi_{t}$ and $\xi_{i}$, to obtain $E_{t}$ and $E_{i}$. The translational energy $E_{t}$ is used to compute the postcollision relative velocity, as described above. The internal energy $E_{i}$ is distributed between the two particles, using degrees of freedom $\xi_{i}^{A}$ and $\xi_{i}^{B}$, to obtain $E_{i}^{A}$ and $E_{i}^{B}$, respectively. The internal energy of particle $A, E_{i}^{A}$, is then distributed between rotational and vibrational modes, with degrees of freedom $\xi_{r}^{A}$ and $\xi_{v}^{A}$, to obtain rotational and vibrational energies, $E_{r}^{A}$ and $E_{v}^{A}$, respectively. The internal energy for particle $B$ is similarly distributed between $E_{r}^{B}$ and $E_{v}^{B}$.

\subsection{Temperature Calculation}

The translational temperature $T_{t}$ for a species is calcualted with,

$$
T_{t}=\frac{m\left(\bar{v}^{2}-\bar{v}^{2}\right)}{3 k}
$$

The rotational temperature $T_{r}$ is similarly computed from the average rotational energy $\bar{E}_{r}$, using,

$$
T_{r}=\frac{2 \bar{E}_{r}}{k \xi_{r}} .
$$

The vibrational temperature $T_{v}$ is computed using the characteristic vibrational temperature $\Theta_{v}$ and the average vibrational energy $\bar{E}_{v}$,

$$
T_{v}=\frac{\Theta_{v}}{\ln \left(1+k \frac{\Theta_{v}}{E_{v}}\right)} .
$$

The total temperature $T$ can then be computed using,

$$
T=\frac{\xi_{t} T_{t}+\xi_{r} T_{r}+\xi_{v} T_{v}}{\xi_{t}+\xi_{r}+\xi_{v}},
$$

where $\xi_{t}=3$ is the number of translational degrees of freedom.

\subsection{Box Tests}

A series of tests was performed to validate this implementatoin of the Larsen-Borgnakke energy exchange model. These were designed to verify correct equilibrium conditions, as well as translational-rotational (TR) and translational-rotational-vibrational (TRV) relaxation rates. These tests were performed for a uniform box with specular walls. 


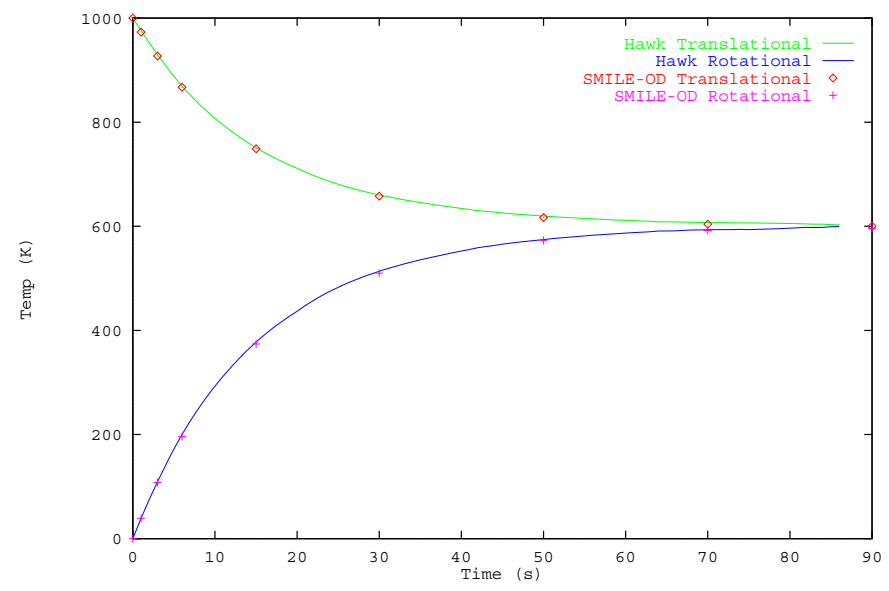

Figure 15: TR Relaxation

The first test was for $\mathrm{N} 2$ at equilibrium in a box, with translational, rotational, and vibrational temperatures equal to $10,000 \mathrm{~K}$, density $1.83 \mathrm{e} 19$ particles per cubic meter, constant TR exchange probability $\phi_{R}=0.2$, and constant TRV exchange probability $\phi_{V}=0.02$. The system stayed in equilibrium for $\alpha=0,0.5$ and 0.24 .

The next case considered relaxation between translational and rotational modes for $\alpha=0.24$. Initially, the translational temperature was $1000 \mathrm{~K}$, the rotational temperature $0 \mathrm{~K}$, and the vibrational temperature $0 \mathrm{~K}$. The TR exchange probability was constant at $\phi_{R}=0.2$, and no TRV exchanges took place $\left(\phi_{V}=0\right)$. The results for Hawkand SMILE, agree as shown in Figure 15.

The next case considered relaxation between translational, rotational, and vibrational modes, with parameters as shown in the table below.

\begin{tabular}{|l|r|r|}
\hline Species & $\mathrm{N} 2$ & \\
\hline$\alpha$ & 0.24 & \\
\hline$\beta$ & 0.735 & \\
\hline$\phi_{R}$ & 0.2 & \\
\hline$\phi_{V}$ & 0.02 & \\
\hline$\theta_{v}$ & $3390 \mathrm{~K}$ & $\mathrm{~K}$ \\
\hline$T_{r e f}$ & $273 \mathrm{~K}$ & $\mathrm{~K}$ \\
\hline$\sigma_{r e f}$ & $5.3068 \times 10^{-19}$ & $m^{2}$ \\
\hline$T_{t}$ & 10000 & $\mathrm{~K}$ \\
\hline$T_{r}$ & 5000 & $\mathrm{~K}$ \\
\hline$T_{v}$ & 0 & $\mathrm{~K}$ \\
\hline
\end{tabular}

The next case was a mixture of Nitrogen and Oxygen, each with internal degrees of freedom, 


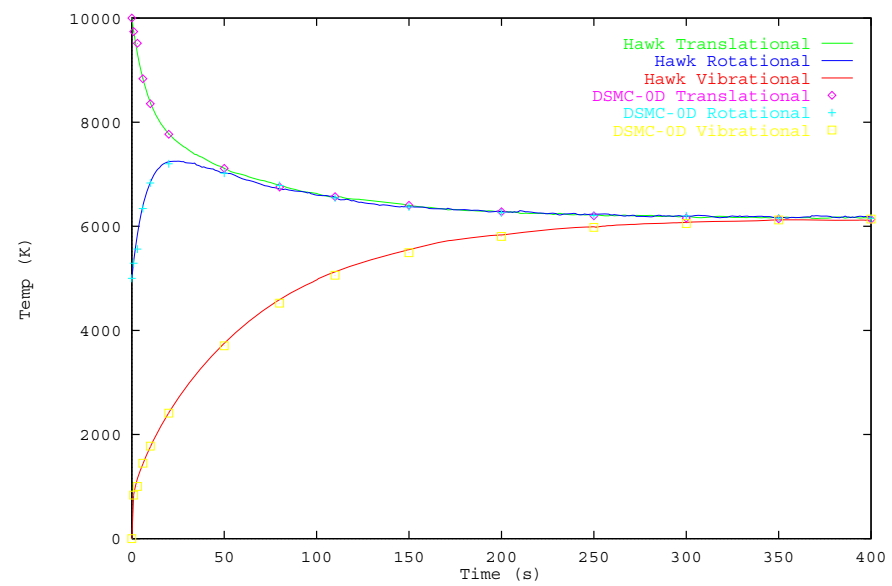

Figure 16: TRV Relaxation

and with TR and TRV exchange probablilties as specified in the table below.

\begin{tabular}{|l|r|r|r|l|}
\hline Species & N2-N2 & N2-O2 & O2-O2 & \\
\hline$\alpha$ & 0.24 & 0.255 & 0.27 & \\
\hline$\beta$ & 0.735 & 0.725 & 0.7143 & \\
\hline$\phi_{R}$ & 0.2 & 0.2 & 0.2 & \\
\hline$\phi_{V}$ & 0.02 & 0.02 & 0.02 & \\
\hline$\theta_{v}$ & 3390 & & 2256 & $\mathrm{~K}$ \\
\hline$T_{\text {ref }}$ & 273 & 273 & 273 & $\mathrm{~K}$ \\
\hline$\sigma_{\text {ref }}$ & $5.3068 \times 10^{-19}$ & $5.3069 \times 10^{-19}$ & $5.307 \times 10^{-19}$ & $m^{2}$ \\
\hline Fraction & .5 & & .5 & \\
\hline $\mathrm{Tt}$ & 10000 & & 15000 & $\mathrm{~K}$ \\
\hline $\mathrm{Tr}$ & 5000 & & 7500 & $\mathrm{~K}$ \\
\hline $\mathrm{TV}$ & 0 & & 0 & $\mathrm{~K}$ \\
\hline
\end{tabular}

As shown in Figure 17, agreement was excellent between Hawkand SMILE.

The next test considered variable exchange probabilities, $\phi_{R}(T)$ and $\phi_{V}(T)$. As shown in Figure 18, the results agreed very well with SMILE.

\subsection{Cylinder Test}

The final Larsen-Borgnakke test was for supersonic flow around the cylinder. Figure 19 shows a comparison of temperature profiles between Hawkand SMILE. Figures 20 and 21 show translational temperature flowfields for the two codes. Results agree to within statistical scatter. 


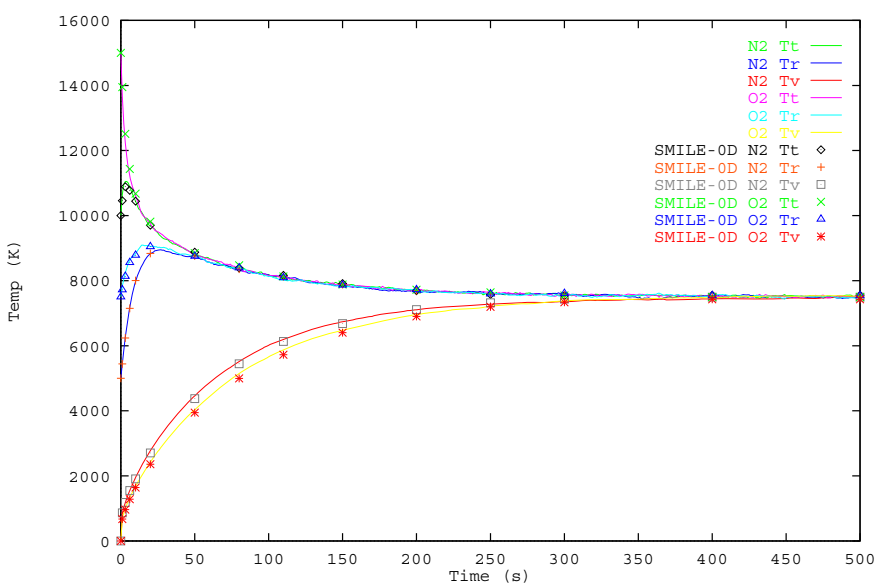

Figure 17: TRV Mixture

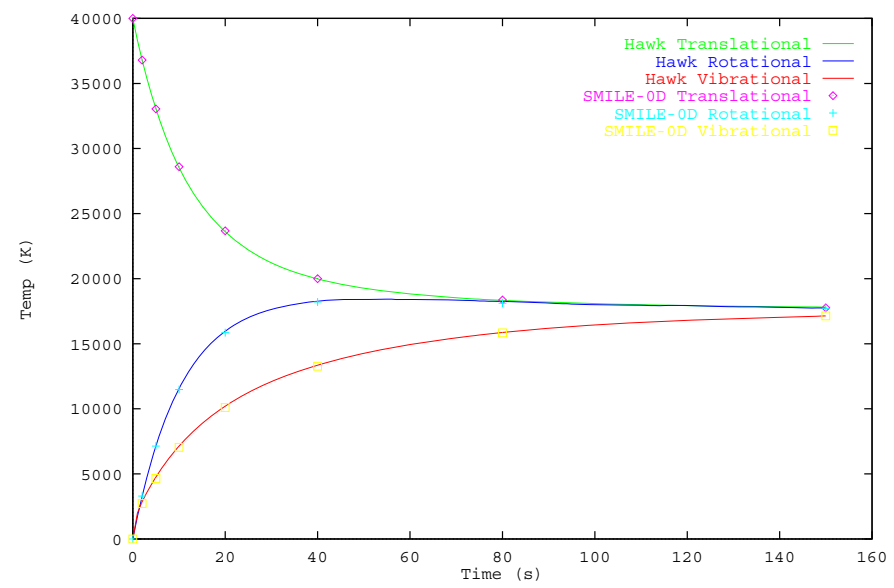

Figure 18: Variable $\phi_{R}, \phi_{V}$ 


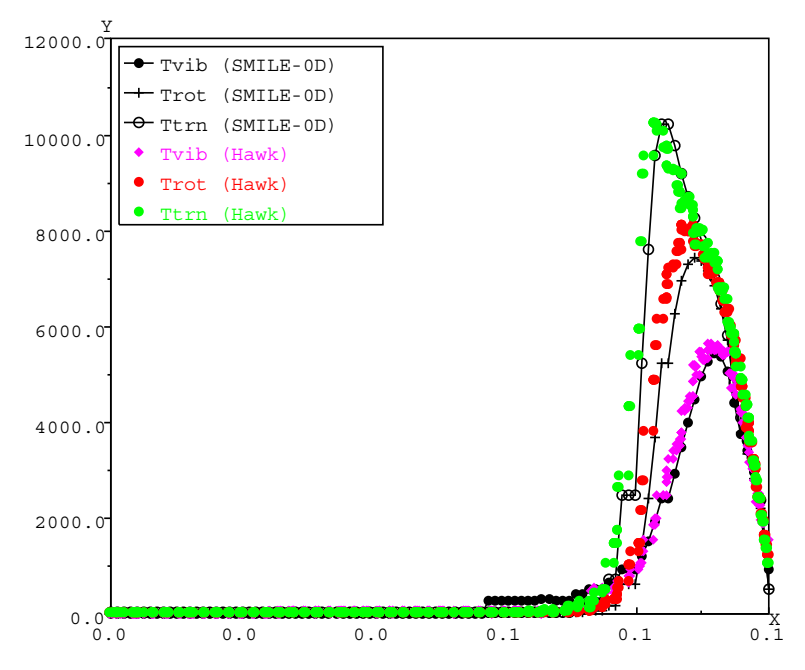

Figure 19: TRV Cylinder

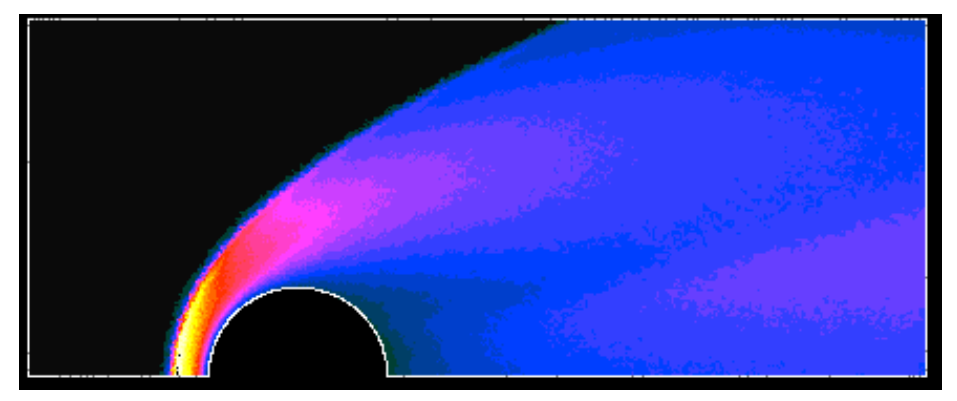

Figure 20: HawkTranslational Temperature

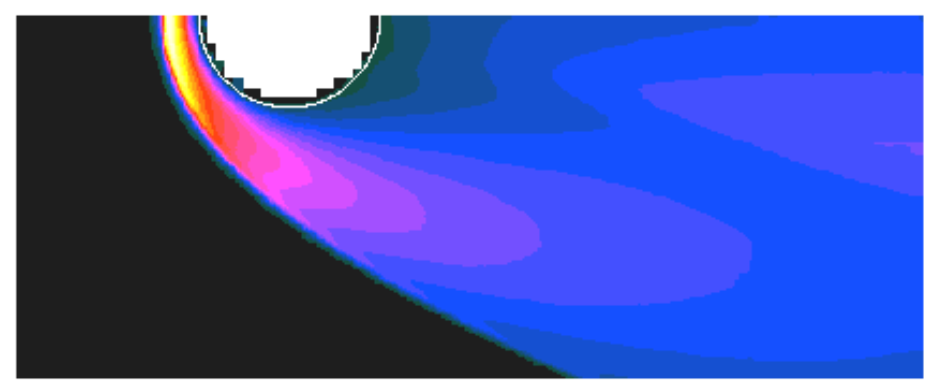

Figure 21: SMILE Translational Temperature 


\section{General Collision Algorithm}

This section describes the algorithm for computing all of the collisions in a given cell. The variable recompute is used to detect when the collision frequencies must be recomputed. This is necessary during the first iteration of the loop, as well as any time a reaction takes place. The total collision frequency $\nu$ is the sum of the collision frequencies $\nu_{i j}$ between all pairs of species $i$ and $j$.

1. recompute $=$ True

2. $\mathrm{t}=0$

3. while $t<$ timestep

(a) if recompute

i. let $\nu=\sum_{i j} \nu_{i j}$

ii. recompute $=$ False

(b) $\tau=\frac{-\ln \left(R_{1}\right)}{\nu}$

(c) $t=t+\tau$

(d) if $t<$ timestep

i. Select species $i, j$

ii. Select particles $a, b$

iii. if $\frac{\sigma_{i j}\left(g_{a b}\right) g_{a b}}{\left[\sigma_{i j} g\right]_{\text {max }}}>R_{2}$
A. Perform Collision
B. If reaction, recompute $=$ True

The collision frequency between species $i$ and $j$ is computed using

$$
\nu_{i j}=\left\{\begin{array}{ll}
\frac{N_{i}\left(N_{i}-1\right) F_{N}\left[\sigma_{i j} g\right]_{\max }}{2 V} & i=j \\
\frac{N_{i} N_{j} F_{N}\left[\sigma_{i j} g\right]_{\max }}{V} & i \neq j
\end{array},\right.
$$

where $\left[\sigma_{i j} g\right]_{\max }$ is the maximum value of $\sigma_{i j}\left(g_{i j}\right)$ that has been observed between any pair of colliding particles $i$ and $j, F_{N}$ is the ratio of real to simulated particles, and $V$ is the volume of 
the cell. Note that a separate value of $\left[\sigma_{i j} g\right]_{\max }$ is kept for each pair of species. The species $i$ and $j$ are chosen with probability $\frac{\nu_{i j}}{\nu}$. The particles $a$ and $b$ are selected randomly from the lists for species $i$ and $j$.

Once two particles have been selected for a collision, the collision takes place according to the following algorithm. A collision may be reacting or non-reacting. Each possible reacting collision, or reaction, $k$, has an associated cross section, $\sigma_{k}(g)$, and will occur with probability $\frac{\sigma_{k}(g)}{\sigma_{t}(g)}$. The sum of $\sigma_{k}$ over all reactions is less than or equal to the total cross section of the collision, $\sigma_{t}$. Not all collisions, therefore, are reacting. If a reaction takes place, internal energy is exchanged as described in Section 7.3.

1. Select reaction $k$ with probability $\frac{\sigma_{k}(g)}{\sigma_{t}}$.

2. If reaction $k$ selected
(a) Perform reaction $k$
(b) It will be necessary to recompute $\nu$ and $\nu_{i j}$.

3. Else if no reaction selected

(a) Exchange internal energy if necessary.

(b) Perform collision

4. Update particle velocities and species

If no reaction is selected, an elastic or inelastic collision takes place. With probability $P_{T R}$, a TR exchange will take place. With probability $P_{T R V}$, a TRV exchange will take place. If neither occurs, a elastic collision will be performed. If a reaction is selected, the energy of the reaction $E_{k}$ is used to compute the post-collision energy $E_{c}^{\prime}$ from the pre-collision energy $E_{c}, E_{c}^{\prime}=E_{c}-$ $E_{k}$. The particles are then moved to the new species lists, and the post collision velocities are computed. 


\section{Conclusion}

The HawkDSMC implementation now contains several collision models, Hard Sphere (HS), Variable Hard Sphere (VHS), Variable Soft Sphere (VSS), and Larsen-Borgnakke. The motivation, implementation, and validation of each of these models has been presented. Results show that the implementation is correct, in comparison with another DSMC implementation.

The tools described in this paper are currently in use by Intel and Tegal Corporations for design and evaluation of plasma reactors, and by the Institute for Defense Analyses for reentry calculations. Future work will include implementation and validation of more sophisticated reactions, such as dissociation and recombination. Reactions on surfaces will also be addressed, once adequate models are available. Once these additional features are in place, reliable, accurate, and detailed reactor simulations will be possible. These in turn will facilitate the design of the next generation of plasma reactors.

\section{Acknowledgements}

The authors would like to gratefully acknowledge the contributions of Evan Cohn, Xavier Fan, Bob Haimes, Mikhail Ivanov, Alexander Kashkovsky, John Maweu, Bradley Nelson, Karie Smart, Stephen Taylor, and Jerrell Watts. The research described in this report is sponsored by Intel Corporation and the Advanced Research Projects Agency under contract number DABT63-95C-0116. The information contained herein does not necessarily reflect the position or policy of the government of the United States, and no official endorsement should be inferred. Access to the Cray T3D was provided by JPL/NASA, and access to the Intel Paragon was provided by Caltech/CACR.

\section{References}

[Bird94] G. Bird. "Molecular Gas Dynamics and the Direct Simulation of Gas Flows." Clarendon Press. Oxford, 1994.

[Borgnakke75] C. Borgnakke, P. Larsen. "Statistical Collision Model for Monte Carlo Simulation of Polyatomic Gas Mixture.” J. Comp. Phys. (18) 1975. 
[Ivanov92] M. Ivanov, S. Antonov, S. Gimelshein, A. Kashkovsky. "Rarefied Numerical Aerodynamic Tools for Reentry Problems." Proc. I Europ. Comp. Fluid Dyn. Conf., Burssels, Belgium. 1992.

[Ivanov94] M. Ivanov, S. Antonov, S. Gimelshein, A. Kashkovsky. "Computational Tools for Rarefied Aerodynamics." Proc. XVII Intern. symp. on Rarefied Gas Dynamics, Vancouver, Canada. 1994.

[Lumpkin91] F. Lumpkin III, B. Haas, I. Boyd. "Resolution of the differences between collision number definitions in particle and continuum simulations." Phys. Fluids A. 3(9). 1991.

[Maweu96] J. Maweu, M. Rieffel. "The SCP Grid Library." Caltech Technical Report CS96-07. 1996.

[Millikan63] R. Millikan, D. White. "Systematics of Vibrational Relaxation." J. Chem. Phys. 39(12). 1963.

[Nanbu81] K. Nanbu, Y. Watanabe. "Relaxation Rates of Inverse-Power and Rigid-Sphere Molecules." Rep. Ins. High Speed Mach.. (43) 334.1981

[Park85] C. Park. "Problems of Rate Chemistry in the Flight Regimes of Aeroassisted Orbital Transfer Vehicles." Progr. in Astronautics and Aeronautics, Vol. 96, New York. 1985.

[Parker59] J. Parker. "Rotational and Vibrational Relaxation in Diatomic Gases." Phys. Fluids. 2(4). 1959.

[Rieffe195] M. Rieffel. "Concurrent Simulations of Plasma Reactors for VLSI Manufacturing." Caltech Masters Thesis CS-95-012. 1995.

[Taylor96] S. Taylor, J. Watts, M. Rieffel, M. Palmer. "The Concurrent Graph: Basic Technology for Irregular Problems." IEEE Parallel and Distributed Technology. 1996.

[Zhong95] X. Zhong, K. Koura. "Comparison of Solutions of the Burnett Equations, Navier-Stokes Equations, and DSMC for Couette Flow." Rarefied Gas Dynamics 19. Volume 1, Oxford University Press. 1995. 